\title{
Cerebrospinal Fluid Otorrhea
}

National Cancer Institute

\section{Source}

National Cancer Institute. Cerebrospinal Fluid Otorrhea. NCI Thesaurus. Code C34461.

Discharge of cerebrospinal fluid through the ear structures. 